\title{
ORIGINAL ARTICLE \\ Successful tracheostomy decannulation after complete or sensory incomplete cervical spinal cord injury
}

\author{
DH Kim ${ }^{1}$, SW Kang ${ }^{2}$, WA Choi ${ }^{2}$ and $\mathrm{HJ} \mathrm{Oh}^{2}$
}

Study design: Retrospective study.

Objectives: To report the successful tracheostomy decannulation/extubation in cervical spinal cord injury (C-SCI) patients.

Setting: Tertiary university hospital, pulmonary rehabilitation center.

Methods: Complete or sensory incomplete C-SCI patients who had received invasive acute phase respiratory management, and succeeded in decannulation/extubation became candidates of this study. Retrospective review was conducted on the transitions of the respiratory status.

Results: Sixty-two patients (M: 55, F: 7) were identified. Traumatic etiologies accounted for 93.5\%, and mean onset age was $47.6 \pm 15.8$ years. Sixty patients $(96.8 \%)$ had undergone tracheostomy and the other two received endotracheal intubation during acute phase. All patients succeeded in decannulation/extubation after employing mechanically assisted coughing and noninvasive mechanical ventilation (NIV). Mean time since tracheostomy to decannulation was $7.0 \pm 14.5$ months. Of the 60 tracheostomized patients, 12 succeeded in decannulation without applying long-term NIV, 31 switched to continuous NIV after decannulation. Fifteen patients totally weaned off from ventilators after NIV. Two patients who once succeeded in decannulation were re-tracheostomized. For the 31 patients with continuous NIV, mean hours of daily need for ventilatory support had reduced from $15.3 \pm 8.0$ to $5.7 \pm 5.7 \mathrm{~h}$ at final follow-ups.

Conclusion: Complete or sensory incomplete C-SCl patients, even with high neurological level can be successfully decannulated through NIV and aggressive use of mechanically assisted coughing. Undesirable tracheostomy can be avoided by employing the noninvasive respiratory management.

Spinal Cord (2017) 55, 601-605; doi:10.1038/sc.2016.194; published online 24 January 2017

\section{INTRODUCTION}

Cervical spinal cord injury (C-SCI) accounts for an important cause of disability in trauma patients. Besides motor paralysis, respiratory complications are still the leading cause of morbidity and mortality in individuals with C-SCI. ${ }^{1}$ Approximately two-thirds of patients experience respiratory complications, such as atelectasis, pneumonia and ventilatory failure during acute phase, which may require mechanical ventilation and tracheostomy. ${ }^{2,3}$ The degree of respiratory dysfunction is closely related to the extent and level of the neurological injury. ${ }^{2}$ Injuries above the level of phrenic motor neurons $(\mathrm{C} 3,4,5)$ cause both diaphragm and expiratory muscle paralysis, ${ }^{1}$ thereby leading to inspiratory failure and inadequate clearance of secretions. Consequently, individuals with a complete injury or high neurological level (NL) are exposed to a higher risk of devastating respiratory complications.

It has been reported that $21-77 \%$ of patients with C-SCI have undergone tracheostomy. ${ }^{4}$ Tracheostomy provides a secure airway, facilitates airway secretion control ${ }^{4}$ and early tracheostomy may decrease pulmonary septic complications in trauma patients. ${ }^{5}$ Although controversy remains, early tracheostomy placement had been advocated from the perspective of reducing the overall medical costs in the acute phase. ${ }^{4-8}$ Several previous reports exist which reveal its beneficial effect in facilitating ventilator management and weaning, ${ }^{6}$ decrease duration of intensive care unit stay ${ }^{8}$ and reduce acute hospital admission time. ${ }^{4,6}$

Although tracheostomy and tracheostomy mechanical ventilation (TMV) had been regarded essential in the long-term management of C-SCI patients, common complications associated with long-term TMV, such as tracheal stenosis, tracheomalacia and stomal infection, cannot be overlooked. ${ }^{9}$ Evidences are being accumulated on noninvasive acute and long-term management of respiratory failure even to the high C-SCI patients. ${ }^{9-11}$ Noninvasive measures include tracheostomy decannulation, continuous ventilatory support by noninvasive positive pressure ventilation to support weakened inspiratory muscles and mechanically assisted coughing (MAC) to support both in-expiratory muscles. ${ }^{11}$ By employing the noninvasive management, complications associated with tracheostomy as well as the need for invasive airway suctioning and uncomfortable tube changes can be avoided, which consequently results in fewer hospitalizations, less pulmonary morbidity and lower cost in the long term. ${ }^{12,13}$

In this study, we aimed to review the successful decannulation or extubation for patients with complete or sensory incomplete

${ }^{1}$ Department of Rehabilitation Medicine, Kangdong Sacred Heart Hospital, Hallym University College of Medicine, Seoul, Korea and ${ }^{2}$ Department of Rehabilitation Medicine, Pulmonary Rehabilitation Center, Gangnam Severance Hospital, Rehabilitation Institute of Neuromuscular Disease, Yonsei University College of Medicine, Seoul, Korea Correspondence: Professor SW Kang, Department of Rehabilitation Medicine, Pulmonary Rehabilitation Center, Gangnam Severance Hospital, Yonsei University College of Medicine, 211 Eunju-ro, Gangnam-gu, Seoul 06273, Korea.

E-mail: kswoong@yuhs.ac.

Received 2 June 2016; revised 4 December 2016; accepted 18 December 2016; published online 24 January 2017 
C-SCI who had received either tracheostomy or endotracheal intubation for respiratory management during the acute phase. We compared the respiratory status of tetraplegic C-SCI patients before the initial admission and outcomes of the same patients after being treated at our pulmonary rehabilitation center.

\section{MATERIALS AND METHODS}

\section{Subjects}

Individuals with complete or sensory incomplete C-SCI who were referred to pulmonary rehabilitation center at the Gangnam Severance hospital in a state of tracheostomy or intubation, and succeed in either decannulation or extubation from March 2003 to December 2015 became candidates of this study. Sixty-two C-SCI patients were identified during the study period. Mean onset age was $47.6 \pm 15.8$ years. Traumatic SCI accounted for the majority of etiologies (93.5\%), mostly because of traffic accidents or fall downs. Proportions of high C-SCI (NL C4 or above) was remarkably high (90.3\%), and also the number of patients with complete injury was higher (79.0\%).

Medical charts, including discharge summaries, imaging studies and detailed pulmonary function test results, were individually reviewed retrospectively. Parameters analyzed included patient demographics (age, sex and onset age), mechanism of injury, admission American spinal injury association (ASIA) grade of C-SCI, NL, presence of other associated traumatic injuries, pre-morbid lung disease (such as tuberculosis, chronic obstructive lung disease and asthma) and types of acute respiratory care status before admission, such as intubation, tracheostomy or use of mechanical ventilation. Transitions of respiratory care status and subjects' final respiratory status after noninvasive management at our center were compiled from medical records.

\section{Decannulation and/or transition to noninvasive ventilation}

Decannulation/extubation criteria were as follows: alert and fully cooperative, no apparent lung diseases, no dysphagia, all oxyhemoglobin saturation $\left(\mathrm{SpO}_{2}\right)$ $\geqslant 95 \%$ at ambient air and airway patency with intact vocal cord movement. Patients with concomitant lung disease, bulbar-innervated muscle dysfunction and lack of cooperation were excluded from decannulation/extubtion attempts.

The presence of dysphagia was evaluated with videofluoroscopic swallowing study. All of the tracheostomized patients underwent videofluoroscopic swallowing study on their first admission, and those without apparent aspiration or penetration with liquid and semisolid consistency were included. Flexible fiber optic endoscopy was used to evaluate the airway patency under consultation to ENT specialist. Tracheostomy tubes were switched to cuffless ones with two different inner cannulas (fenestrated and non-fenestrated) for

\section{Table 1 General characteristics of all patients}

\begin{tabular}{ll}
\hline Onset age (years) & $47.6 \pm 15.8$ \\
Sex (M:F) & $55: 7$ \\
Duration from tracheostomy to decannulation (months) & $7.0 \pm 14.5$ \\
Neurological level (n) & \\
C- & 1 \\
C1 & 3 \\
C2 & 9 \\
C3 & 23 \\
C4 & 20 \\
C5 & 2 \\
C6 & 2 \\
C7 & 0 \\
C8 & 2 \\
ASIA impairment scale (n) & \\
A & 49 \\
B & 13 \\
Etiologies of injury & \\
Traumatic & 58 \\
Non-traumatic & 4 \\
\hline Abbreviation: ASIA, American Spinat Iniuny Association.
\end{tabular}

Abbreviation: ASIA, American Spinal Injury Association. alternative use. For patients who fulfilled the above criteria, decannulation attempt was initiated with capping of the tracheostomy tube. Continuous TMV was replaced to noninvasive ventilation (NIV) via nasal or oronasal interfaces depending on each patient's preference. Basically, volume assist/ control mode ventilation was used with tidal volumes individually adjusted to maintain $15-20 \mathrm{cmH}_{2} \mathrm{O}$ of peak inspiratory pressures, and normal physiologic back-up rates for age. Tracheal suctioning was substituted to self-oral expectoration by using mechanical insufflation-exsufflation (MI-E), (CoughAssist (Philips-Respironics International Inc.)) as well as manually assisted cough by abdominal thrust. All patients were trained to MAC, and applied it whenever necessary or at least in every $2 \mathrm{~h}$ to prevent secretion retention.

Detailed decannulation procedures followed the sequence proposed by Bach et al. ${ }^{9,11,14}$ If decannulation was not feasible during the first admission, patients and care givers were sufficiently trained to various assisted coughing methods and NIV before home discharge. When patients were ready and fulfilled the above criteria, they were re-admitted to complete the decannulation procedure.

\section{Respiratory and ventilatory function assessment}

Suitability of ventilatory status was monitored by $\mathrm{SpO}_{2}$ and end tidal carbon dioxide $\left(\mathrm{EtCO}_{2}\right)$ or transcutaneous $\mathrm{CO}_{2}\left(\mathrm{~T}_{\mathrm{c}} \mathrm{CO}_{2}\right)$ via oximetry and capnometry (Microcap plus, Oridion Ltd., Jerusalem, Israel) (V-sign System, SenTec, Therwil, Switzerland) or sometimes with arterial blood gas analysis.

For the pulmonary function assessment, forced vital capacity in two different positions, maximum inspiratory pressure and maximum expiratory pressure were measured periodically to evaluate respiratory muscle strength and weaning potentials. To assess each patient's capability of self-expectoration, peak cough flow was measured under two different conditions: unassisted and assisted with maximal insufflations by air-stacking followed by manual abdominal thrust. All measurements were performed by a peak flowmeter (ASSESS; Health Scan Products Inc., Cedar Grove, NJ, USA) with the patient coughing as hard as possible, and the highest value of three or more attempts was recorded. In this study, we only included the data evaluated within 5 days from decannulation despite serial measurements.

\section{RESULTS}

A total of 62 complete or sensory incomplete C-SCI patients succeed in either extubation or decannulation during the study period. Common co-morbidities at initial admission included pressure ulcers, depression, urinary tract infection, formation of heterotopic ossifications and pulmonary complications such as pneumonia and pleural effusion. Baseline characteristics of all subjects are described in Table 1.

\section{Respiratory management status before initial admission}

All subjects had received endotracheal intubation or tracheostomy during acute phase of SCI. When referred to our center, two NL C4 and ASIA impairment scale (AIS)-A patients were placing endotracheal intubation tubes, and both had been applying continuous mechanical ventilation for the acute respiratory care.

Forty-one patients were under continuous TMV at initial admission to the pulmonary rehabilitation center. Nineteen patients were maintaining tracheostomy tubes after weaning of intensive care unit mechanical ventilators. All of the tracheostomized patients either failed or did not have decannulation attempts during previous hospitalizations. Details of the acute respiratory management status before admission are described in Table 2.

\section{Final respiratory status after treatment at pulmonary rehabilitation} center

Two patients with endotracheal intubation were successfully extubated with the use of MAC, and switched to continuous NIV: one patient 
Table 2 Acute phase respiratory management before initial admission

\begin{tabular}{|c|c|c|c|c|c|}
\hline & \multirow[t]{2}{*}{ Categories } & \multicolumn{3}{|c|}{$N L$} & \multirow[t]{2}{*}{$\mathrm{n}$} \\
\hline & & $C$ - to $C 2$ & C3 to C5 & $\mathrm{C} 6$ to $\mathrm{C} 8$ & \\
\hline 2 & Continuous mechanical ventilation through intubation & 0 & 2 & 0 & 2 \\
\hline \multirow[t]{2}{*}{3} & Tracheostomy status after weaning of ICU or portable ventilators, without applying mechanical ventilation & 1 & 17 & 1 & 19 \\
\hline & Total & 13 & 45 & 4 & 62 \\
\hline
\end{tabular}

Abbreviations: ICU, intensive care unit; NL, neurological level.

Table 3 Final respiratory state of patients at discharge

\begin{tabular}{|c|c|c|c|c|c|}
\hline & \multirow[t]{2}{*}{ Categories } & \multicolumn{3}{|c|}{$N L$} & \multirow[t]{2}{*}{$\mathrm{n}$} \\
\hline & & $C-$ to $C 2$ & C3 to C5 & $\mathrm{C} 6$ to $\mathrm{C} 8$ & \\
\hline 1 & Transition to NIV after extubation/decannulation & 6 & 18 & 1 & 25 \\
\hline 2 & Ventilator weaning after extubation/decannulation & 5 & 10 & 1 & 16 \\
\hline 3 & TMV with re-tracheostomy after decannulation & 1 & 0 & 1 & 2 \\
\hline 4 & Simple decannulation without applying long-term MV & 1 & 10 & 1 & 12 \\
\hline \multirow[t]{2}{*}{5} & Application of NIV after decannulation & 0 & 7 & 0 & 7 \\
\hline & Total & 13 & 45 & 4 & 62 \\
\hline
\end{tabular}

Abbreviations: MV, mechanical ventilation; NL, neurological level; NIV, noninvasive mechanical ventilation; TMV, tracheostomy mechanical ventilation.

succeeded in the weaning of NIV after 3 months of use, and the other one was still applying nocturnal NIV because of sustained hypercapnea.

Of the 41 patients who were initially applying continuous TMV, 24 switched to NIV and were still applying continuous mechanical ventilation with reduced extent of application time. Fifteen patients totally weaned off from NIV after the decannulation. Two C-SCI patients with NL C2 and C8, AIS-A were successfully decannulated, but re-tracheostomized during follow-up period due to severe pneumonia and recurrent airway obstruction with mucus plugging despite aggressive use of MAC.

Nineteen C-SCI patients were in tracheostomy state without applying mechanical ventilation at initial admission. Ten tracheostomized C-SCI patients succeeded in decannulation and never required continuous NIV, and two others succeeded in decannulation and ventilator weaning after employing short-term NIV. However, four C-SCI patients required application of long-term NIV due to latent hypercapnea. Underlying moderate to severe obstructive sleep apnea was discovered in three patients during ventilatory state monitoring, which required application of nocturnal NIV with continuous positive airway pressure or bilevel positive airway pressure mode.

In this present study, high C-SCI with NL at C4 or above accounted for the majority (90.3\%). Among the 62 study candidates, 44 patients were motor and sensory complete (AIS-A) with high-level C-SCI. Ten of them succeeded in decannulation and NIV weaning after short-term use, 7 no longer required NIV after the decannulation and 1 patient underwent re-tracheostomy after a successful decannulation. The other 26 converted to NIV, and continuously applying NIV and MAC without significant pulmonary complications.

Mean follow-up period was $21.3 \pm 29.8$ months. For those who switched to NIV, initial hours of daily need for ventilatory support was $15.3 \pm 8.0 \mathrm{~h}$, but gradually decreased to $5.7 \pm 5.7 \mathrm{~h}$ at final discharge.
Details of final respiratory state of all subjects are summarized in Table 3.

Results of pulmonary function assessment just before decannulation

Serial pulmonary function assessments were conducted during individual patient's hospitalizations. To understand the underlying pulmonary function according to the NL and access the weaning potential, we reviewed the result of pulmonary function evaluation just before each patient's decannulation. On retrospective review, measurement data were available only for the 42 patients. For the 20 patients, the measurement was either incomplete or lacking. Detailed pulmonary function measurement is described in Table 4.

\section{DISCUSSION}

Patients who sustain high C-SCI frequently require ventilatory assistance during acute and rehabilitation periods, and in routine clinical practices, they are often managed with intubation and mechanical ventilation, leading to tracheostomy. Bellamy et al. ${ }^{15}$ reported that $77 \%$ of patients with complete tetraplegia underwent tracheostomy, and one recent analysis revealed $69 \%$ of C-SCI with AIS-A required tracheostomy for pulmonary care, weaning and airway support. ${ }^{6}$

Partial recovery of respiratory muscle performance may occur over the year following injury, resulting in subsequent increase in forced vital capacity. ${ }^{1}$ Nevertheless, many SCI patients who failed to wean or decannulate during acute injury period are often discharged home or long-term facilities with home mechanical ventilation and tracheostomy tubes.

In this present study, we reviewed the successful decannulation cases of complete or sensory incomplete C-SCI patients, $70 \%$ of whom were having high NL and complete injuries. Considering the results from the previous researches regarding tracheostomy and decannulation of 
Table 4 Results of pulmonary function assessment just before decannulation

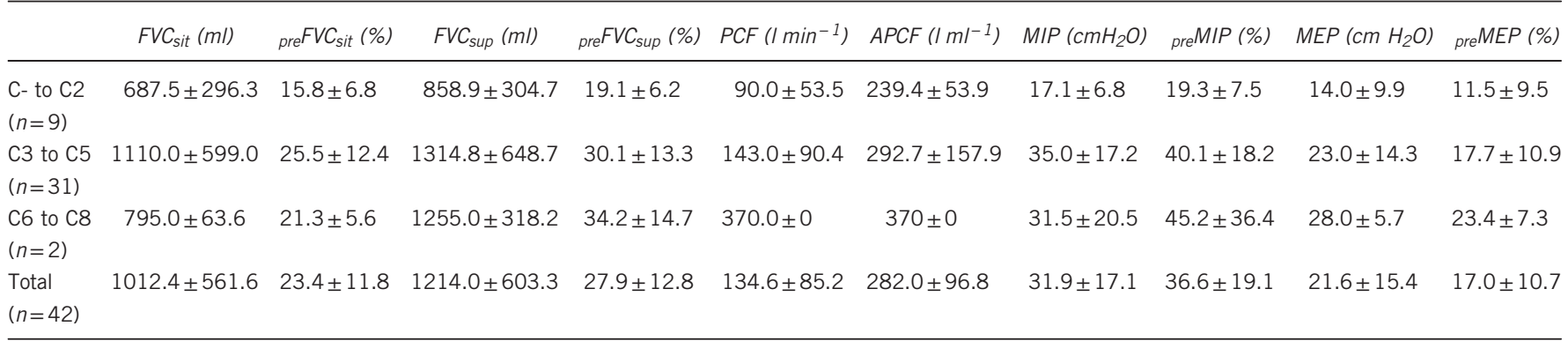

Abbreviations: $\mathrm{APCF}$, assisted peak cough flow; $\mathrm{FVC}_{\text {sit }}$, forced vital capacity in sitting position; pre $\mathrm{FVC}_{\text {sit }}$, normal predicted value of $\mathrm{FVC}_{\text {in }}$ sitting position; $\mathrm{FVC}_{\text {sup }}$, forced vital capacity in supine position; pre $\mathrm{FVC}_{\text {sup, }}$, normal predicted value of $\mathrm{FVC}$ in supine position; MEP, maximum expiratory pressure; MEP pre, normal predicted value of MEP; MIP, maximum inspiratory pressure;

MIP pre, normal predicted value of MIP; PCF, peak cough flow.

All values are mean \pm s.d.

C-SCI patients, our study result has a great significance. Childs et al. ${ }^{16}$ concluded that AIS-A is a simple criterion to identify the need of prolonged mechanical ventilation and the need for tracheostomy, and another study revealed that the NL and completeness of SCI are the most important predictors of tracheostomy. ${ }^{17}$

In previous literatures, success in weaning from mechanical ventilation approached $40 \%$ in patients with cervical injuries above C4, with increasing success in injuries below C5.,18 In this study, successful weaning from TMV or NIV after decannulation was $43.8 \%$ in patients with NLI at or above C4, and $30 \%$ in NL at or below C5. The majority of our candidates were high C-SCI, and only six patients were included for the NLI at or below C5. A small number of the group and variability in durations from C-SCI onset to decannulation might result in the reversed outcome from the previous studies.

Possible complications associated with decannulation include immediate respiratory distress, post-decannulation pneumonia within $24 \mathrm{~h}^{19}$ and sudden mucus plugging, all of which might result in reinsertion of tracheostomy tubes. In this study, two patients with NL C2 and C8, AIS-A were recannulated due to severe pneumonia and recurrent airway obstruction with mucus plugging despite aggressive use of MAC. For decannulation trial, we mainly used cuffless tracheostomy tube with two different inner cannulasfenestrated and non-fenestrated ones for alternative use during a day in order to minimize relevant complications associated with the lengthy use of fenestrated tubes such as tracheal irritation or granulation formation. ${ }^{20}$ During the study period, our patients did not reveal any relevant adverse effect on laryogoscopic evaluations.

Bach $^{11}$ had addressed a new management paradigm to permit a successful extubation of unweanable SCI patients without tracheostomy, and permits patients with ineffective cough to avoid acute respiratory failure due to mucus plugging during respiratory tract infections. Tracheostomy decannulation was even possible for patients with little or no ventilator-free breathing ability and no vital capacity, and the only indication for tracheostomy was SCI patients with saliva aspiration irrespective of optimal use of NIV and MAC. ${ }^{11}$

Noninvasive respiratory management is known to offer several advantages over endotracheal intubations or tracheostomies. Management at our center included air-stacking exercise, manually assisted coughing and the use of MI-E and NIV. The risk of failed extubation or decannulation is high in C-SCI due to pulmonary secretion retention and inadequate cough. MI-E is started by applying positive pressure to the airway (insufflations) to immediately transform into negative pressure (exsufflations) using a mechanical device. ${ }^{2}$ With pressure approximately $\pm 40 \mathrm{cmH}_{2} \mathrm{O}$, it generates an expiratory flow of approximately $101 \mathrm{~s}^{-1}, 21$ which is much higher than the manualassisted cough. ${ }^{22}$ Clinical practice guideline from the Consortium for Spinal Cord Medicine mentions the use of MI-E on respiratory management following SCI. ${ }^{23}$ Aggressive use of MI-E results in immediate expulsion of thick secretions, which is the most problematic in C-SCI, and it also increases in vital capacity and $\mathrm{SpO}_{2} \cdot{ }^{14}$

This study shares the limitation inherent to all retrospective cohorts. Retrospective study design might influence our results. To emphasize the feasibility of successful decannulation for C-SCI with high NL and complete injuries, we only included successful cases which might represent a selection bias. Second, although our center is a nationwide referral center for pulmonary rehabilitation, this study was a single center design. Because of the relatively small number of patients, multi-center based further study should be followed. Third, detailed information on each patient's acute management such as operative procedure, associated injuries at initial onset could not be fully collected. Because of the limited information regarding acute injury period, the analysis of the variables associated to the successful decannulation or ventilator weaning could not be conducted.

In conclusion, tracheostomy decannulation is even possible for the patients with complete or sensory incomplete C-SCI and high level of $\mathrm{NL}$, and noninvasive respiratory intervention, including NIV and $\mathrm{MAC}$, is an effective long-term alternative to tracheostomy. Avoiding the empiric use of tracheostomy may decrease numerous tracheostomy-related complications and respiratory morbidity, and ultimately improve the quality of life in several aspects.

\section{DATA ARCHIVING}

There were no data to deposit.

\section{CONFLICT OF INTEREST}

The authors declare no conflict of interest.

\section{ACKNOWLEDGEMENTS}

This study was supported by a SRC research grant of Yonsei University College of Medicine for (3-2015-0264).

1 Brown R, DiMarco AF, Hoit JD, Garshick E. Respiratory dysfunction and management in spinal cord injury. Respir Care 2006; 51: 853-868.

2 Galeiras Vazquez R, Rascado Sedas P, Mourelo Farina M, Montoto Marques A, Ferreiro Velasco ME. Respiratory management in the patient with spinal cord injury. Biomed Res Int 2013; 2013: 168757.

3 Wong SL, Shem K, Crew J. Specialized respiratory management for acute spinal cord injury: a retrospective analysis. Top Spinal Cord Inj Rehabil 2012; 18: 283-290. 
4 Nakashima H, Yukawa Y, Imagama S, Ito K, Hida T, Machino M et al. Characterizing the need for tracheostomy placement and decannulation after cervical spinal cord injury. Eur Spine J 2013; 22: 1526-1532.

5 Klugger Y, Paul DB, Lucke J, Cox P, Colella JJ, Townsend RN et al. Early tracheostomy in trauma patients. Eur J Emerg Med 1996; 3: 95-101.

6 Harrop JS, Sharan AD, Scheid EH Jr, Vaccaro AR, Przybylski GJ. Tracheostomy placement in patients with complete cervical cord injuries: American Spinal Injury Association Grade A. J Neurosurg 2004; 100: 20-23.

7 Rodriquez JL, Steinberg SM, Luchetti FA, Gibbons KJ, Taheri PA, Flint LM. Early tracheostomy for primary airway management in the surgical critical care setting. Surgery 1990; 108: 655-659.

8 Brook AD, Sherman G, Malen J, Kollef MH. Early versus late tracheostomy in patients who require prolonged mechanical ventilation. Am J Crit Care 2000; 9: 352-359.

9 Bach JR, Alba AS. Noninvasive options for ventilator support of the traumatic high level quadriplegic patients. Chest 1990; 98: 613-619.

10 Roki A, Hanayama K, Ishikawa Y. Resolution of tracheostomy complications by decannulation and conversion to noninvasive management for a patient with highlevel tetraplegia. Top Spinal Cord Inj Rehabil 2012; 18: 193-196.

11 Bach JR. Noninvasive respiratory management of high level spinal cord injury. J Spinal Cord Med 2012; 35: 72-80

12 Bach JR, Rajaraman R, Ballanger F, Tzeng AC, Ishikawa Y, Kulessa R et al. Neuromuscular ventilator insufficiency: the effect of home mechanical ventilator usw vs oxygen therapy on pneumonia and hospitalization rates. Am J Phys Med Rehabil 1998; 77: 8-19.

13 Bach JR, Intintola P, Alba AS, Holland I. The ventilator-assisted individuals: cost analysis of institutionalization versus rehabilitation and in-home management. Chest 1992; 101: 26-30.
14 Bach JR, Saporito LR, Shah HR, Sinquee D. Decannulation of patients with severe respiratory muscle insufficiency: efficacy of mechanical insufflation-exsufflation. J Rehabil Med 2014; 46: 1037-1041.

15 Bellamy R, Pitts FW, Stauffer SE. Respiratory complications in traumatic quadriplegia. Analysis of 20 years' experience. J Neurosurg 1973; 39: 596-600.

16 Childs BR, Moore RA, Como JJ, Vallier HA. American spinal injury association impairment scale predicts the need for tracheostomy after cervical spinal cord injury. Spine 2015; 40: 1407-1413.

17 Arora S, Flower O, Murray NP, Lee BB. Respiratory care of patients with cervical spinal cord injury: a review. Crit Care Resusc 2012; 14: 64-73.

18 Berney S, Bragge P, Granger C, Opdam H, Denehy L. The acute respiratory management of cervical spinal cord injury in the first 6 weeks after injury: a systemic review. Spinal Cord 2011; 49: 17-29.

19 Cohen O, Tzelnick S, Lahav Y, Stavi D, Shoffel-Havakuk H, Hain M et al. Feasibility of a single-stage tracheostomy decannulation protocol with endoscopy in adult patients. Laryogoscope 2016; 126: 2057-2062.

20 Merritt RM, Bent JP, Smith RJ. Suprastomal granulation tissue and pediatric tracheostomy decannulation. Laryngoscope 1997; 107: 868-871.

21 Gomez-Merino E, Sancho J, Marin J, Severa E, Blasco ML, Belda FJ et al. Mechanical insufflations-exsufflation: pressure, volume, and flow relationships and the adequacy of the manufacturer's guidelines. Am J Phys Med Rehabil 2002; 81: 579-583.

22 Bach JR. Mechanical insufflations-exsufflation. Comparison of peak expiratory flows with manually assisted and unassisted coughing techniques. Chest 1993; 104: 1553-1562.

23 Consortium for spinal cord medicine. Respiratory management following spinal cord injury: a clinical practice guideline for health-care professionals. J Spinal Cord Med 2005; 28: 259-293. 